\title{
Development and Application of a Multilocus Sequence Analysis Method for the Identification of Genotypes Within Genus Bradyrhizobium and for Establishing Nodule Occupancy of Soybean (Glycine max L. Merr)
}

\author{
Peter van Berkum, ${ }^{1}$ Patrick Elia, ${ }^{1}$ Qijian Song, ${ }^{1}$ and Bertrand D. Eardly ${ }^{2}$ \\ ${ }^{1}$ Soybean Genomics and Improvement Laboratory, Agricultural Research Service, United States Department of Agriculture, \\ Beltsville, MD 20705, U.S.A., and ${ }^{2}$ Penn State Berks College, Berks Campus, P.O. Box 7009 Reading, PA 19610, U.S.A.
}

Submitted 18 September 2011. Accepted 1 November 2011.

\begin{abstract}
A multilocus sequence typing (MLST) method based on allelic variation of seven chromosomal loci was developed for characterizing genotypes (GT) within the genus Bradyrhizobium. With the method, 29 distinct multilocus GT were identified among 190 culture collection soybean strains. The occupancy of 347 nodules taken from uninoculated field-grown soybean plants also was determined. The bacteroid GT were either the same as or were closely related to GT identified among strains in the culture collection. Double-nodule occupancy estimates of $2.9 \%$ were much lower than values published based on serology. Of the 347 nodules examined, 337 and 10 were occupied by Bradyrhizobium japonicum and $B$. elkanii, respectively. The collection strains within the species $B$. japonicum and $B$. elkanii also were compared with Bradyrhizobium cultures from other legumes. In many cases, the observed GT varied more according to their geographic origin than by their trap hosts of isolation. In other cases, there were no apparent relationships with either the legume or geographic source. The MLST method that was developed should be a useful tool in determining the influence of geographic location, temperature, season, soil type, and host plant cultivar on the distribution of GT of Bradyrhizobium spp.
\end{abstract}

After corn (Zea mays L.), soybean (Glycine max L. Merr) is the most important agricultural crop in the United States, valued at $\$ 29.6$ billion in the 2008 to 2009 growing season. Most of the crop is processed to produce soybean meal for animal feed and oil that is increasingly used in the manufacture of biodiesel. Commercial fertilizer is applied up to $40 \%$ of the soybean acreage, even though the crop is a legume and can benefit from a nitrogen-fixing symbiosis with rhizobia. Appropriate

Corresponding author: P. van Berkum; Telephone: +1.301 .504 .7280 ; Fax: +1.301.713.0308; E-mail: peter.vanberkum@ars.usda.gov

Nucleotide sequence data is available in the GenBank database under the following accession numbers: alleles of asd, JN685821 to JN685872; alleles of gapA, JN685873 to JN685927; alleles of $\operatorname{gyrB}$, JN685725 to JN685772; alleles of ilvI, JN685928 to JN685979; alleles of lepA, JN685773 to JN685820 ; alleles of $m d h$, JN685980 to JN686029; alleles of purC, JN686030 to JN686088.

This article is in the public domain and not copyrightable. It may be freely reprinted with customary crediting of the source. The American Phytopathological Society, 2012. rhizobial cultures can be applied to soybean at the time of sowing (inoculation) to encourage nitrogen input from biological nitrogen fixation.

The correlation between inoculation leading to formation of nodules on soybean roots and improved plant performance was well established as early as the late 1800 s, long before this legume became an important crop in the United States (Brooks 1901; Cotrell et al. 1900, Hopkins 1904). The value of inoculating soybean subsequently stimulated research that attempted to establish relationships between the rhizobia of soybean and other legumes. At first, these early studies principally depended upon cultural characteristics and host plant infection (Carroll 1934; Leonard 1923; Sears and Carroll 1927) but the lack of reliability motivated development of serology for species identification as used with animal pathogens. As early as 1932, the existence of several serological groupings among soybean strains had been revealed (Fred et al. 1932), culminating in the description of 17 different serogroups by 1965 (Date and Decker 1965).

Serology became an important approach in the research of the soybean-microbe interaction because the soybean-nodulating rhizobia exhibit a high degree of serological specificity, with polyclonal antibodies prepared against somatic antigens. For example, serological methods have been used extensively in the study of symbiosis, inoculation success, and rhizobial ecology (Berg et al. 1988; Boonkerd et al. 1978; Caldwell 1969; Caldwell and Hartwig 1970; Caldwell and Vest 1968; Caldwell and Weber 1970; Cregan and Keyser 1986; Damirgi et al. 1967; Ellis et al. 1984; Fuhrmann 1990; Ham et al. 1971; Johnson et al. 1965; Kapusta and Rouwenhorst 1973; Keyser and Cregan 1987; Kvien et al. 1981; Means et al. 1964; Mpepereki and Wollum 1991; Schmidt et al. 1968; Thompson et al. 1991; Vest et al. 1971; Weber et al. 1989; Yokoyama et al. 1999).

A significant problem with serology was a high incidence of multiple reactions with occupants of a single nodule (Lindemann et al. 1974; Moawad and Schmidt 1987; Mpepereki and Wollum 1991). Nodules with several different serologically reactive occupants were initially thought to be the cause of the multiple reactions (Lindemann et al. 1974; Moawad and Schmidt 1987). However, Mpepereki and Wollum (1991) reported that nodules with multiple serological reactions represented a single occupant with a distinctive serology rather than a mixture of different serological individuals. Consequently, the effectiveness of serology in monitoring soybeanrhizobia interaction appears limited, which would justify the development of an alternative approach that probably would best 
be based on a method using molecular biology. In developing an alternative method, consideration should be given to the extensive literature originating from research using serology.

Molecular methods were used on a smaller scale for the purpose of taxonomy. This led to the separation of a few soybean rhizobial strains into the species Bradyrhizobium japonicum (Jordan 1982) and B. elkanii (Kuykendall et al. 1992). However, there have been few reports linking the serology of multiple soybean rhizobial strains with results obtained using molecular methods. An investigation of sequence divergence in the $16 \mathrm{~S}$ rRNA gene and the internally transcribed spacer (ITS) between the $16 \mathrm{~S}$ and $23 \mathrm{~S}$ rRNA genes separated $B$. japonicum serogroups $4,6,38,62,110,122,123,124,126,127,129$, and 135 from B. elkanii serogroups 31, 46, 76, 94, and 130 (van Berkum and Fuhrmann 2000). However, using the 16S rRNA gene for the study of plant-microbe interaction in the soybean symbiosis would be impractical because of the limited sequence divergence among members of the serogroups within each of the two species. Although the ITS between the $16 \mathrm{~S}$ and $23 \mathrm{~S}$ rRNA genes was more variable (van Berkum and Fuhrmann 2000), the reported sequence identity in this region across different members within each serogroup (van Berkum and Fuhrmann 2009) would limit its usefulness. Consequently, the development of an alternative approach would be necessary to compare results obtained by a molecular analysis with the extensive literature based on serology.

In two recent reviews, the merits of using a multilocus molecular analysis for genotyping members within different rhizobial species was discussed (Eardly and van Berkum 2005; van
Berkum and Eardly 2005). In this approach, the alleles of at least seven chromosomal loci are identified by direct nucleotide sequence comparisons (Maiden et al. 1998). A combination of the alleles for each of the loci examined in a collection of individual cultures is then used to describe an allelic profile or the genotype (GT) of each strain. The data obtained by this method, known as multilocus sequence typing (MLST), are maintained in a database that can be manipulated to permit subsequent comparisons of large numbers of GT without the need to repeatedly analyze reference cultures. MLST results for numerous pathogenic bacterial species (Feil 2004) and Medicago spp.-nodulating rhizobia (van Berkum et al. 2006, 2007, 2010) have already been reported. However, this strategy has never been applied to the rhizobia of soybean to link serological affinities and chromosomal GT.

The aim of the present work was to i) examine the relationships between multilocus chromosomal GT and the 17 serogroups that have been previously established in soybean, ii) determine whether certain chromosomal GT are associated with specific legume hosts or geographic origins, and iii) establish whether MLST can be effectively used to determine nodule occupancy of soybean plants grown at a field site having an extensive history of studies based on serology.

\section{RESULTS}

Analysis of culture collection soybean strains.

All of the polymerase chain reaction (PCR) primer pairs that were designed for the seven loci were used in amplification

Table 1. Primers used for polymerase chain reaction (PCR) and sequence analysis of seven chromosomal loci in genomes of rhizobia in the genus Bradyrhizobium

\begin{tabular}{|c|c|c|c|}
\hline Locus $^{\mathrm{a}}$ & Primer sequence & Length sequenced (bp) & Locus location in Bradyrhizobium japonicum USDA 110 \\
\hline \multicolumn{4}{|l|}{ asd } \\
\hline PCR:33U21 & CGGCAATGTCGGACGSGAAAT & 434 & 536968 to 538002 \\
\hline PCR:604L21 & TGTAGCCGTCCTYCATGAAGA & $\ldots$ & $\ldots$ \\
\hline seq:41U21 & TCGGACGSGAAATGCTCAACA & $\ldots$ & $\ldots$ \\
\hline seq:508L21 & GGCCTTGGTCTGCGAGAACAA & $\ldots$ & $\ldots$ \\
\hline \multicolumn{4}{|c|}{ 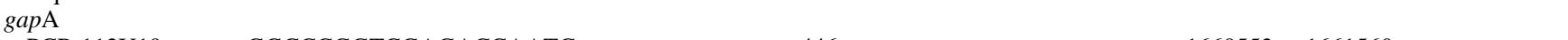 } \\
\hline PCR:112U19 & GGCCCGGTCGAGACCAATG & 446 & 1660553 to 1661560 \\
\hline PCR:957L20 & CGGCCATGCGGTTCGAGAAG & $\ldots$ & $\ldots$ \\
\hline seq:477U21 & GCCGRTCGCCAAGGTSCTGAA & $\ldots$ & $\ldots$ \\
\hline seq:937L21 & GCCCCACTCRTTGTCGTACCA & $\ldots$ & $\ldots$ \\
\hline \multicolumn{4}{|c|}{ - } \\
\hline PCR:105U21 & CAAGCGCCCGGGCATGTATAT & 438 & 4814257 to 4816302 \\
\hline PCR:855L21 & TGCGGGATGTTGTTGGTGAAG & $\ldots$ & $\ldots$ \\
\hline seq:141U21 & CGGCTCSGGCCTGCACCACAT & $\ldots$ & $\ldots$ \\
\hline seq:658L19 & CGTGGCGCATGTCGGAGAG & $\ldots$ & $\ldots$ \\
\hline \multicolumn{4}{|c|}{ 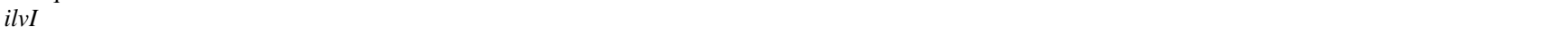 } \\
\hline PCR:942U23 & YCCGTCCTCGATCAACAAGAACA & 375 & 7158294 to 7158836 \\
\hline PCR:1675L23 & TGCGCCTTGCCSGASGGGATCAT & $\ldots$ & $\ldots$ \\
\hline seq:1056U21 & CAAGGCGTGGTGGCAGCAGAT & $\ldots$ & $\ldots$ \\
\hline seq:1664L21 & AGGGGATCATCGGGAAGCAGT & $\ldots$ & $\ldots$ \\
\hline \multicolumn{4}{|c|}{ 19 } \\
\hline PCR:291U21 & CACGCCCGGCCAYGTCGACTT & 434 & 8947810 to 8949660 \\
\hline PCR:1322L17 & GCGGCGGTCCTGGCACA & $\ldots$ & $\ldots$ \\
\hline seq:411U21 & CCAGGCGCTCGACAACAATCA & $\ldots$ & $\ldots$ \\
\hline seq:956L21 & CGTCGGCGTCGACCGGGAACA & $\ldots$ & $\ldots$ \\
\hline \multicolumn{4}{|l|}{$m d h$} \\
\hline PCR:3U20 & GGCGCGCGACAAGATTGCTT & 451 & 494219 to 495187 \\
\hline PCR:898L18 & CACSGCGSCSACCGACTT & $\ldots$ & $\ldots$ \\
\hline seq:309U21 & CGGCGCCGGCATCAAGAAGTA & $\ldots$ & $\ldots$ \\
\hline seq:799L21 & MACGCCGACATACATGTCCTT & $\ldots$ & $\ldots$ \\
\hline \multicolumn{4}{|l|}{ purC } \\
\hline PCR:110U19 & GAGGGCAAGGGYGTCCTCA & 394 & 870860 to 871774 \\
\hline PCR:504L20 & CCCGCACTCCATCTTGAAGT & $\ldots$ & $\ldots$ \\
\hline seq:134U23 & CGGATYTCGGAGTACCTGTTTCA & $\ldots$ & $\ldots$ \\
\hline seq:474L19 & ATGCCGATGCCGAGGAAGA & $\ldots$ & $\ldots$ \\
\hline
\end{tabular}


reactions with the FailSafe PCR buffer E (Epicenter, Madison, WI, U.S.A.) producing amplified segments that were 571, 845, 750, 733, 1,031, 895, and 394 bp for asd, gapA, gyrB, ilvI, lepA, $m d h$, and purC, respectively. The length sequenced for each of these loci ranged from 375 to $451 \mathrm{bp}$ (Table 1).

After developing and testing the efficiency of the method with United States Department of Agriculture (USDA) 110 ( $B$. japonicum) and USDA 76 (B. elkanii), it was applied in the analysis of 190 soybean strains of the USDA Agricultural Research Service (ARS) National Rhizobium Germplasm Collection, a significant number of which could be assigned to $B$. japonicum or B. elkanii by serological affinity. These 190 strains were divided into 29 separate GT based on variation in the alleles of the seven loci (Table 2). Of the 17 different serogroups, 15 were shown to have unique GT (Table 2). Exceptions were the reference strains of serogroup 31 (USDA 31) and serogroup 127 (USDA 127), which had the same GT as the reference strains of serogroup 76 (USDA 76) and serogroup 123 (USDA 123), respectively. Among the 29 GT, 21 and 8 were identified with B. japonicum (GT-1, GT-4 through GT-15, GT20 through GT-22, GT-24 through GT-26, GT-28, and GT-29) and $B$. elkanii (GT-2, GT-3, GT-16 through GT19, GT23, and GT-27), respectively. Also, the number of strains within the same GT varied from 1 to 21 (Table 2).

\section{Analysis of Bradyrhizobium cultures from host legumes other than soybean.}

Strains representing seven additional Bradyrhizobium spp. (Fig. 1) as well as both B. japonicum and B. elkanii, were analyzed to determine whether the MLST method could be successfully applied to a wide range of Bradyrhizobium cultures that originated from a variety of geographic locations and leg- ume hosts (Table 3). The sequences of the alleles present in the chromosome of each strain were concatenated and then aligned $(2,960 \mathrm{bp})$ to derive a NeighborNet diagram intended to portray the genotypic relationships among the different chromosomes (Fig. 1). The analysis included a reference for each of the 29 GT identified among the culture collection soybean strains. A network was used to display these relationships because a statistically significant probability for recombination was indicated $\left(P\right.$ value $\left.=4.20 \times E^{-8}\right)$ using the pairwise homoplasy index test (Bruen et al. 2006). Rectangles within the network diagram reflect conflicting phylogenetic signals in segments of the aligned sequence data, which may be interpreted as evidence of recombination.

\section{MLST analysis of bacteroids}

to determine nodule occupancy.

During the development of the protocol to extract bacteriod DNA directly from nodules, it was observed that it was necessary to initially remove small plant particles from the suspensions to avoid a significant reduction in the yield of the PCR amplification. This step also helped to reduce nonspecific interference during the subsequent sequence analysis. The debris removal was accomplished by allowing plant particles to settle for a few minutes prior to siphoning off the bacteroids and placing them in a fresh microcentrifuge tube for the remainder of the extraction.

The five soybean plants examined yielded 148 and 199 tap and lateral root nodules, respectively. Double occupancy by two distinct GT was shown in $10(2.9 \%)$ of the nodules. Some minor variation in the distribution of the GT in the tap and lateral root nodules was noted; however, the differences were not considered to be significant (Fig. 2). The majority of the nod-

Table 2. Multilocus sequence typing analysis of 190 United States Department of Agriculture (USDA) ARS Rhizobium Culture Collection soybean strains with genotype (GT) designation and allelic profiles of the seven chromosomal loci ${ }^{\mathrm{a}}$

\begin{tabular}{|c|c|c|c|c|c|c|c|c|c|}
\hline \multirow[b]{2}{*}{ Allelic profile } & \multirow[b]{2}{*}{ Strain } & \multirow[b]{2}{*}{$N^{\mathbf{b}}$} & \multicolumn{7}{|c|}{ Loci } \\
\hline & & & asd & gap & gyrB & $i l v I$ & lepA & $m d h$ & purC \\
\hline GT-1 & USDA $110^{c}$ & 21 & 1 & 1 & 1 & 1 & 1 & 1 & 1 \\
\hline GT-2 & USDA $76^{c}$ & 21 & 2 & 2 & 2 & 2 & 2 & 2 & 2 \\
\hline GT-3 & USDA $130^{c}$ & 1 & 2 & 2 & 2 & 2 & 2 & 2 & 11 \\
\hline GT-4 & USDA $6^{\mathrm{c}}$ & 6 & 3 & 4 & 3 & 4 & 4 & 9 & 4 \\
\hline GT-5 & USDA $62^{c}$ & 3 & 4 & 1 & 1 & 6 & 6 & 3 & 8 \\
\hline GT-6 & USDA $126^{c}$ & 3 & 4 & 1 & 1 & 8 & 6 & 3 & 8 \\
\hline GT-7 & USDA 445 & 1 & 4 & 1 & 7 & 10 & 10 & 6 & 1 \\
\hline GT-8 & USDA 427 & 6 & 4 & 1 & 7 & 10 & 10 & 6 & 3 \\
\hline GT-9 & USDA 454 & 4 & 4 & 1 & 7 & 10 & 10 & 16 & 1 \\
\hline GT-10 & USDA 440 & 3 & 4 & 1 & 7 & 20 & 10 & 6 & 13 \\
\hline GT-11 & USDA $129^{c}$ & 5 & 4 & 11 & 7 & 10 & 10 & 6 & 3 \\
\hline GT-12 & USDA $4^{\mathrm{c}}$ & 6 & 5 & 3 & 4 & 3 & 3 & 11 & 9 \\
\hline GT-13 & USDA $123^{c}$ & 19 & 6 & 25 & 5 & 9 & 7 & 4 & 6 \\
\hline GT-14 & USDA $122^{c}$ & 8 & 7 & 8 & 9 & 8 & 10 & 3 & 5 \\
\hline GT-15 & USDA $124^{\mathrm{c}}$ & 4 & 8 & 10 & 6 & 13 & 9 & 5 & 18 \\
\hline GT-16 & USDA $46^{c}$ & 12 & 9 & 6 & 12 & 2 & 11 & 8 & 11 \\
\hline GT-17 & USDA 90 & 4 & 9 & 15 & 13 & 15 & 12 & 13 & 11 \\
\hline GT-18 & USDA 61 & 1 & 9 & 15 & 13 & 15 & 12 & 13 & 16 \\
\hline GT-19 & USDA $94^{c}$ & 9 & 10 & 7 & 19 & 7 & 13 & 10 & 25 \\
\hline GT-20 & USDA $135^{\mathrm{c}}$ & 13 & 11 & 12 & 8 & 12 & 8 & 12 & 7 \\
\hline GT-21 & USDA 3 & 20 & 12 & 13 & 11 & 14 & 4 & 9 & 10 \\
\hline GT-22 & USDA 57 & 3 & 12 & 17 & 16 & 4 & 4 & 9 & 4 \\
\hline GT-23 & USDA 33 & 1 & 13 & 16 & 14 & 16 & 15 & 14 & 26 \\
\hline GT-24 & USDA 43 & 1 & 14 & 20 & 15 & 17 & 16 & 63 & 15 \\
\hline GT-25 & USDA $38^{c}$ & 6 & 15 & 5 & 10 & 5 & 5 & 7 & 14 \\
\hline GT-26 & USDA 59 & 2 & 16 & 19 & 17 & 18 & 4 & 17 & 20 \\
\hline GT-27 & USDA 369 & 4 & 17 & 18 & 18 & 19 & 13 & 15 & 27 \\
\hline GT-28 & USDA 444 & 2 & 18 & 1 & 7 & 10 & 10 & 6 & 19 \\
\hline GT-29 & USDA 489 & 1 & 19 & 12 & 8 & 21 & 14 & 18 & 24 \\
\hline
\end{tabular}

a Numbers below each of the genes indicate the identification numbers used for each allele that was revealed.

${ }^{b}$ Number of strains with the identical allelic profile defining the GT.

${ }^{c}$ Reference strain of the serogroup; note that USDA 31 (reference for the 31 serogroup) has the same GT as USDA 76 (reference for the 76 serogroup) and USDA 127 (reference for the 127 serogroup) has the same GT as USDA 123 (reference for the 123 serogroup). 
ule occupants (those of 217 nodules) were GT previously identified as those of the reference cultures for the 110,126, and 123 serogroups: GT-1, GT-6, and GT-13, respectively. Besides these, GT-2, GT-3, GT-4, GT-5, GT-14, and GT-25 were additional nodule occupants identified as of reference strains for the $76,130,6,62,122$, and 38 serogroups (Table 2 ).

Only 12 of the 46 GT revealed among the nodule occupants (Fig. 2, GT shown across the $x$ axis) had previously been identified with the analysis of strains in the collection. However, these 12 GT were observed to occupy 265 of the 347 nodules taken from the field-grown plants. (Fig. 2). The relationships of the remaining 34 GT with each of the 29 GT identified in the collection were inferred from a phylogenetic network obtained from aligned concatenated sequences of alleles of each of the seven loci. The majority of the 34 GT were placed with the GT of the reference strains for the 123,6 , and 110 serogroups (Fig. 3). Based on clustering within the phylogenetic network, 151 nodule occupants were serogroup 110 or 110-like, 127 were serogroup 123 or 123-like, and 57 were serogroup 6 or 6-like. This summary accounted for the occupancies of 335 of the 347 nodules. A further 10 nodules were occupied by GT that were either 76 or 76 serogroup-like. Only two GT (GT-31 and GT-36), identified as B. japonicum, associated with two nodules were not grouped with any of the GT of the soybean culture collection strains (Fig. 3). Of the 347 nodules examined, 337 and 10 were occupied by B. japonicum and $B$. elkanii, respectively.

\section{DISCUSSION}

\section{MLST analysis and serology of soybean strains of the culture collection.}

In the first part of this study, the MLST method was applied to soybean strains that are part of the Rhizobium culture collection maintained at the USDA laboratory in Beltsville, MD, U.S.A. The objective was to compare the chromosomal GT and corresponding serology of the strains. Date and Decker (1965) used 28 strains from the Rhizobium Collection at Beltsville to define 17 different serogroups among the soybean rhizobia. Serology has also been applied to extracted soybean bacteroids as a method to determine nodule occupancy (Means et al. 1964). In the current study, 190 soybean strains, including most of those used by Date and Decker (1965), were separated into 29 distinct GT. The difference in the number of strains examined between the two studies can be explained by the limited number of cultures available at the time of the first study. At the time Date and Decker (1965) developed serology for the characterization of soybean strains, the collection maintained 121 accessions, compared with 436 that are currently available. The MLST analysis as a method to characterize soy-

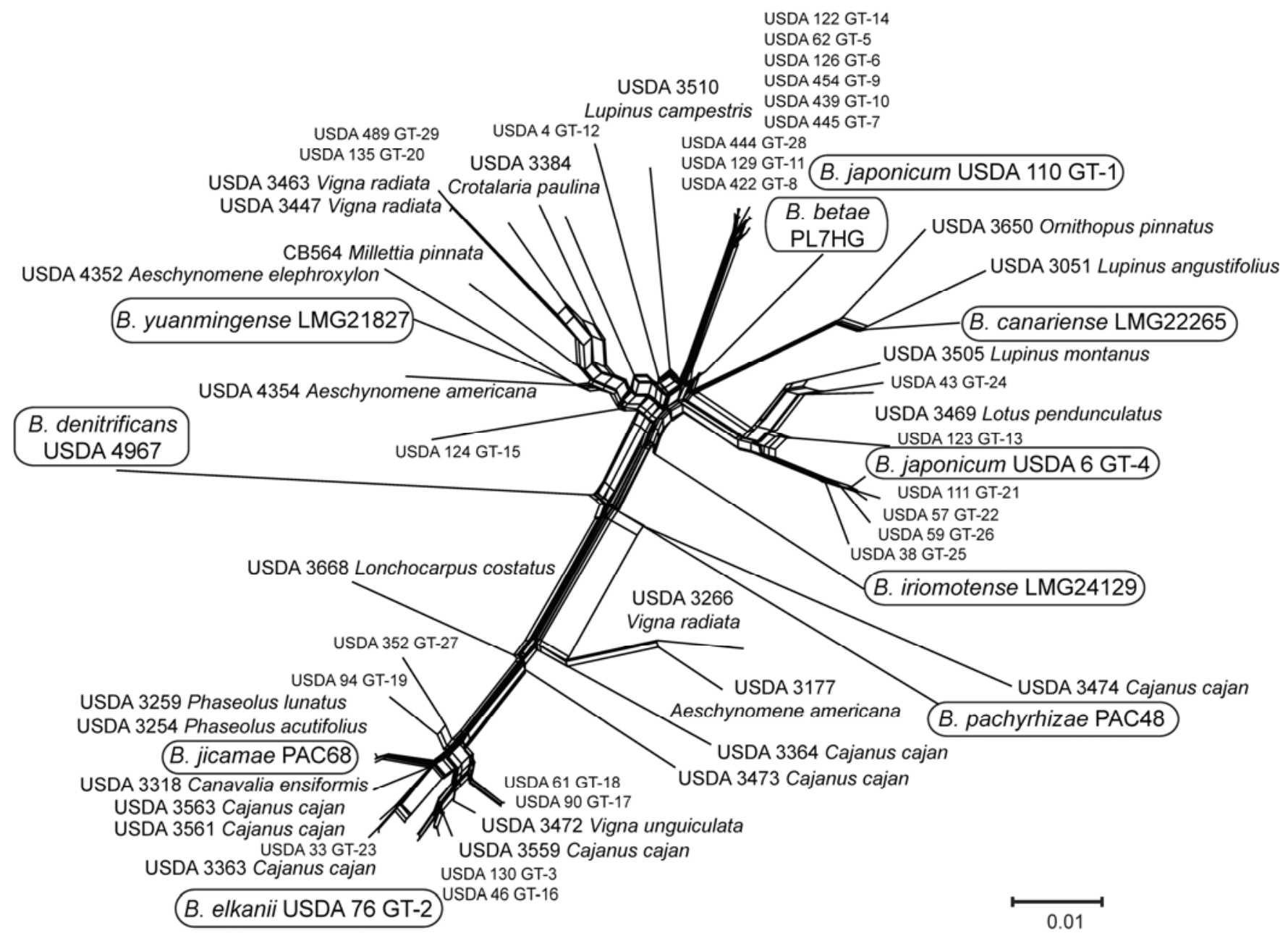

Fig. 1. Estimated evolutionary relationships among strains of Bradyrhizobium originating from different host legume plants and geographic locations. Sequences of seven chromosomal loci were concatenated to produce an alignment of 2,960 bp used in Splitstree version 4.10 (Huson and Bryant 2006) to display evolutionary relationships of the chromosomes as the network algorithm NeighborNet (Bryant and Moulton 2004). A significant probability for recombination among the various chromosomes was shown $\left(P\right.$ value $\left.=4.20 \times E^{-8}\right)$ using the pairwise homoplasy index test $($ Bruen et al. 2006). Aligned sequences of United States Department of Agriculture (USDA) 3384 (host Crotalaria paulina, Brazil), USDA 3451 (host Macrotyloma africanum, Zimbabwe), USDA 3187 (host Arachis hypogea, Zimbabwe), and USDA 3456 (host Vigna unguiculata, Wisconsin, United States) were identical. 
bean strains was more sensitive than serology because seven additional GT were identified among the original 121 accessions used by Date and Decker (1965). However, distinct GT were revealed in only 15 of the 17 reference strains for each of the serogroups that were used to define the serology of the soybean strains.

A problem with serology is that an individual strain may react strongly with more than one antiserum. To overcome this problem, Date and Decker (1965) devised a scheme to determine "serotypes" within serogroups that involved multiple determinations with adsorbed antisera. For example, they identified that, within serogroup 6 , the strain USDA 6 had a serotype distinct from the serogroup 6 strains USDA 3, USDA 24, and USDA 44 by selectively adsorbing antisera prepared against each of the four strains. This protocol made the practical application of serology so complex that, in subsequent studies, the presence of serotypes within each serogroup was ignored. The advantage of MLST is that these serotypes within serogroups probably can be identified because USDA 6 (GT-4) was shown to be distinct from USDA 3 and USDA 24 (both GT-21). Strain USDA 44 was not included in the MLST investigation. In addition to serogroup 6, distinct GT were detected among strains of serogroups $31,94,110,123$, and 135 .

The 123 serogroup is the only example in which selectively adsorbed antisera have been used to distinguish the serotypes of USDA 123, USDA 127, and USDA 129 (Keyser and Cregan 1987; Schmidt et al. 1986). Both USDA 127 and USDA 129 cross-reacted with antisera prepared with USDA 123 but each could be distinguished using its own antiserum that was previously adsorbed using USDA 123 cells. The GT of USDA 123 and USDA 127 could not be distinguished by sequence analysis of the seven loci used in the MLST. Also, the ribosomal region of these two strains cannot be distinguished (van Berkum and Fuhrmann 2009). Consequently, complete genome sequence analyses may be needed with strains USDA 123 and USDA
127 to further resolve their GT. In contrast to USDA 127, the GT of USDA 123 and USDA 129 were distinguishable. The GT of USDA 129 (GT-11) was less divergent with the GT of USDA 110 (GT-1) than it was with the GT of USDA 123 (GT13). A similar genetic relationship between the strains USDA 110, USDA 123, USDA 127, and USDA 129 was reported with results from sequence analysis of the ITS region between the $16 \mathrm{~S}$ and 23S rRNA genes (van Berkum and Fuhrmann 2009).

\section{Comparative analysis of Bradyrhizobium GT of diverse origin.}

An advantage of the approach used in developing MLST was the wide applicability of the method to characterize members of the genus Bradyrhizobium other than those that originated from a symbiosis with soybean. Therefore, it was possible to compare the collection of B. japonicum and B. elkanii with strains isolated from other legume host plants. This was accomplished by concatenating the sequences of the seven alleles within each of the chromosomes and then portraying the relationships with a NeighborNet diagram. From this analysis, it was apparent that, in some cases, a strain's GT was more strongly associated with geographic origin than it was with the trap host. For example, the chromosomes of the two Aeschynomene americana strains USDA 3177 from Arizona and USDA 4345 from Senegal were widely divergent. Similarly, the chromosomes of the Vigna radiata USDA 3266 from Virginia and the two originating from Thailand (USDA 3447 and USDA 3463) also were widely divergent. However, in other cases, neither the host legume nor geographic origin appeared to be related to GT. For example, the chromosomes of three Cajanus cajan strains from Nigeria (USDA 3363, USDA 3561, and USDA 3563) were almost identical to those of several strains of B. elkanii isolated from U.S. soybean. These patterns do not appear to be the result of widespread inoculation practices. Most of the culture collection strains

Table 3. Bradyrhizobium strains used in the multilocus sequence typing analysis that originated from legumes other than Glycine max

\begin{tabular}{|c|c|c|c|}
\hline Host legume of origin & Geographic location of origin & Strain identification number & Reference or source $^{a}$ \\
\hline Aeschynomene americana & Arizona, United States & USDA 3177 & USDA, 1948 \\
\hline A. americana & Senegal & USDA 4354 & Alazard 1985 \\
\hline A. elaphroxylon & Senegal & USDA 4352 (ORS304) & Alazard 1985 \\
\hline Arachis hypogea & Zimbabwe & USDA 3187 & USDA, 1966 \\
\hline Cajanus cajan & India & USDA 3474 & USDA \\
\hline C. cajan & Nigeria & USDA 3363 & USDA, 1975 \\
\hline C. cajan & Bahamas & USDA 3364 & USDA, 1946 \\
\hline C. cajan & India & USDA 3473 & USDA \\
\hline C. cajan & Hawaii & USDA 3559 & USDA, 1962 \\
\hline C. cajan & Nigeria & USDA 3561 & USDA, 1975 \\
\hline C. cajan & Nigeria & USDA 3563 & USDA, 1975 \\
\hline Canavalia ensiformis & Brazil & USDA 3318 & USDA, 1974 \\
\hline Crotalaria paulina & Brazil & USDA 3384 (32H1) & USDA, 1967 \\
\hline Lonchocarpus costatus & Brazil & USDA 3668 (BR6011) & EMBRAPA \\
\hline Lotus pendunculatus & New Zealand & USDA 3469 (NZP2309) & USDA \\
\hline L. uliginosus & unknown & USDA 3470 & USDA \\
\hline Lupinus angustifolius & Georgia, United States & USDA 3051 & USDA, 1946 \\
\hline L. montanus & Mexico & USDA 3505 (FN 13) & E. Martinez-Romero \\
\hline L. campestris & Mexico & USDA 3510 (CICS 70) & E. Martinez-Romero \\
\hline Macrotyloma africanum & Zimbabwe & USDA 3451 (CB756) & USDA, 1960 \\
\hline Milletia pinnata & Australia & CB564 & Norris 1959 \\
\hline Ornithopus pinnatus & New Zealand & USDA 3650 & ICMP \\
\hline Phaseolus acutifolius & Arizona, United States & USDA 3254 & USDA, 1948 \\
\hline P. lunatus & Illinois, United States & USDA 3259 & USDA, 1940 \\
\hline Vigna radiata & Virginia, United States & USDA 3266 & USDA, 1931 \\
\hline V. radiata & Thailand & USDA 3447 & USDA, 1974 \\
\hline V. radiata & Thailand & USDA 3463 & USDA, 1974 \\
\hline V. unguiculata & Wisconsin, United States & USDA 3456 (176A22) & USDA, 1966 \\
\hline V. unguiculata & India & USDA 3472 (P132) & USDA \\
\hline
\end{tabular}

a. USDA = accession in the United Stated Department of Agriculture (USDA) Agricultural Research Service National Rhizobium Resource Collection and year of acquisition given, if known; EMBRAPA = Empresa Brasileira de Pesquisa Agropecuária; and ICMP = International Collection of Micro-organisms from Plants, Auckland, New Zealand. 
were acquired either before inoculation of these crops was practiced (for example, V. radiata, Phaseolus lunatus, and $P$. acutifolius) or were from plants that are not inoculated because they are wild legumes of no agricultural significance (for example, the Aeschynomene spp., Macrotyloma africanum, and Milettia pinnata).

\section{Application of MLST for the determination of nodule occupancy.}

The MLST analysis was successfully applied for the determination of nodule occupancy in uninoculated field-grown soybean. Nodule occupancy in field-grown soybean at the same location in Beltsville had previously been established using serology. Based on results of serology, the 110 and 125 serogroups were the predominant nodule occupants (Boonkerd et al. 1978; Caldwell and Vest 1968; Caldwell and Weber 1970; Johnson et al. 1965). Using MLST as the method of analysis, these would correspond to GT-1 and GT-6 which, in this study, similarly were predominant nodule occupants together with GT-13. The 123 serogroup (GT-13) was not included in the studies using serology, probably explaining why it was not identified among the nodule occupants of soybean at Beltsville. Serogroups 6, 31, 46, 76, 94, and 122 also were reported at lower frequencies among nodule occupants of soybean in the fields at Beltsville (Boonkerd et al. 1978; Caldwell and Vest 1968; Caldwell and Weber 1970; Johnson et al. 1965) and similarly were identified by MLST in this study. It is probably important to point out that a considerable number of nodule occupants could not be identified using serology either because bacteroid preparations cross-reacted with more than one antiserum or did not cross-react at all because of an undefined serology (Boonkerd et al. 1978; Caldwell and Vest 1968; Caldwell and Weber 1970; Johnson et al. 1965). The determination of nodule occupancy by MLST was successful in each case in which the bacteroid DNA extraction had been successful.

\section{Double or multiple nodule occupancy.}

The estimation of double or multiple nodule occupancy using serology is a significant problem and, because of this, the estimates have varied widely. Evidence for multiple nodule occupancy was inferred when bacteroid preparations crossreacted with more than one single antiserum. Reported values of double or multiple nodule occupancy in soybean have ranged from 12 to $54 \%$ (Lindemann et al. 1974; Moawad and Schmidt 1987; Mpepereki and Wollum 1991). However, the cross-reaction of a single bacteroid preparation with several different antisera was shown to be an inherent limitation of serology as the method of analysis rather than an indicator of multiple nodule occupancy. Mpepereki and Wollum (1991) demonstrated this by obtaining single colony isolates from bacteroid preparations that cross-reacted with more than one antiserum and showing that these pure cultures still maintained their multiple serological affinity. They concluded that these bacteroids had a single unique serology and that the original results were not due to a mixture of serological types within a single nodule. This explanation would be supported by the much lower estimate of $2.9 \%$ double occupancy obtained by determining the GT of the bacteroids by MLST analysis in this

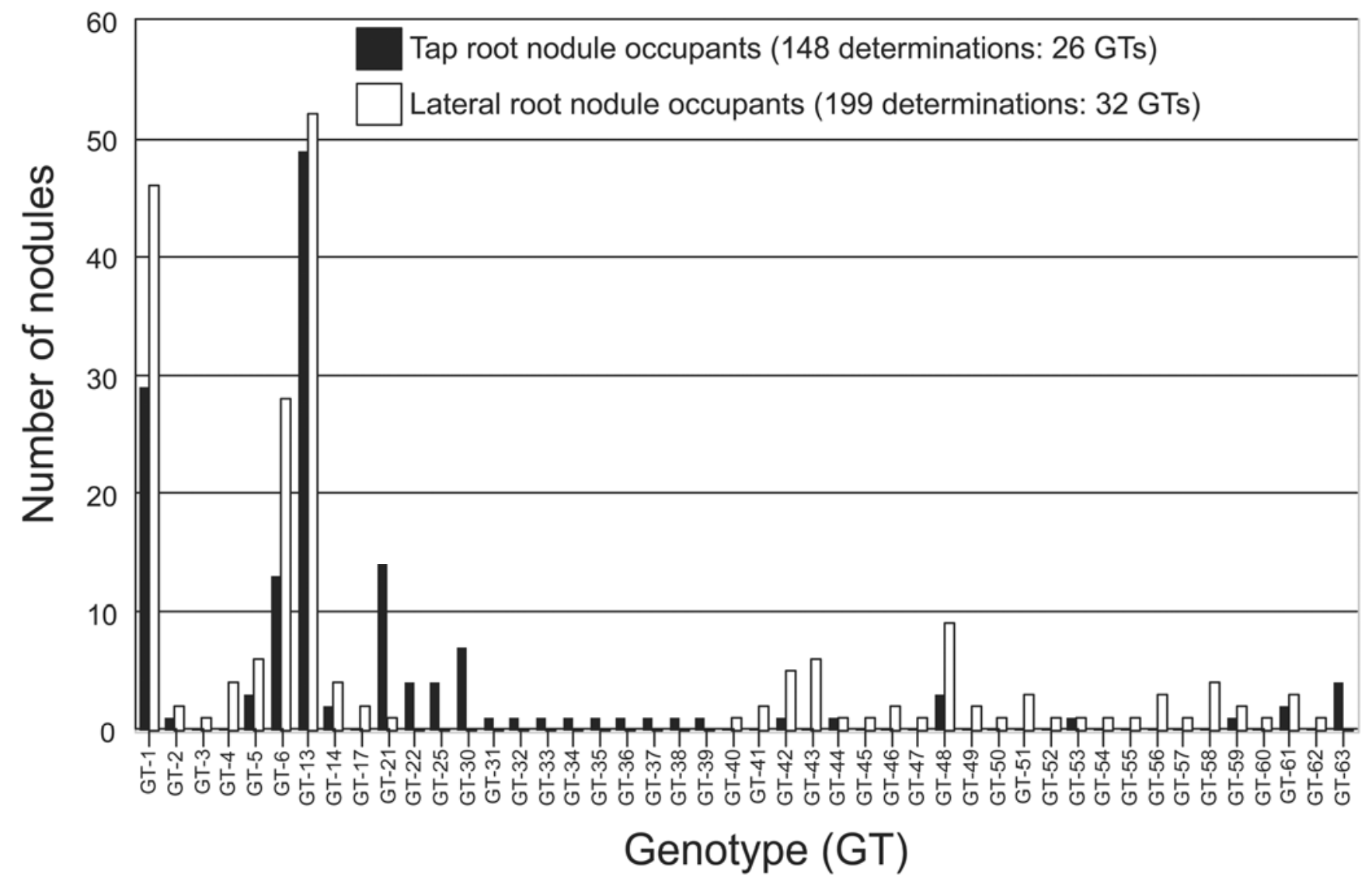

Fig. 2. Number of nodules that were occupied by each of 46 different bacteroid genotypes. Nodules taken from uninoculated soybean (Glycine max L. Merr) Roundup-ready NK Brand S49-Q9 sown for crop production in the South Farm of the Beltsville Agricultural Research Center during summer 2008 were separated into those originating from the tap or lateral roots before preparing bacteroid suspensions for DNA extraction. In all, 12 of the 46 different genotypes shown along the $x$ axis that were revealed by the analysis of bacteroids extracted from 347 nodules previously had been identified as present among the 190 culture collection strains. 
study. The MLST analysis used in this study has been shown to have several advantages over the use of serology and was successfully applied to distinguish culture collection soybean strains and nodule occupants of field-grown soybean and compare soybean strains with strains of other host legumes.

\section{Limitations of serology that can be addressed using MLST.}

The main hypothesis explored in this study was that a straightforward relationship exists between the serogroup and GT of rhizobia that nodulate soybean. The goal was to use this information to provide a means to link data acquired by serology with GT and also to group the soybean rhizobia and other members of the genus Bradyrhizobium. Although evidence was acquired for agreement between serogroup and GT, the relationships were more complex than initially anticipated. In addition to the drawback that the serology for a significant number of the strains is uncertain or not available, more GT than serogroups were identified. Serology was used in previous studies of the symbiosis to determine inoculation success and to investigate rhizobial ecology (Berg et al. 1988; Boonkerd et al. 1978; Caldwell 1969; Caldwell and Hartwig 1970; Caldwell and Vest 1968; Caldwell and Weber 1970; Cregan and Keyser 1986; Damirgi et al. 1967; Ellis et al. 1984; Fuhrmann 1990; Ham et al. 1971; Johnson et al. 1965; Kapusta and Rouwenhorst 1973; Keyser and Cregan 1987; Kvien et al. 1981; Means et al. 1964; Mpepereki and Wollum 1991; Schmidt et al. 1968; Thompson et al. 1991; Vest et al. 1971; Weber et al. 1989) because it was the most appropriate method for distinguishing differences. However, multiple cross-reactions (Date and Decker 1965), the presence of undefined serogroups (Mpepereki and Wollum 1991), and low compatibility between the serology of U.S. strains and those isolated in Southeast Asia (Thompson et al. 1991; Yokoyama et al. 1999) are significant limitations. These limitations are addressed with the use of MLST to determine GT. The method not only can be applied to soybean strains that have low compatibility with antisera raised against the 17 serogroups (Thompson et al. 1991; Yokoyama et al. 1999) but also facilitates the comparison of

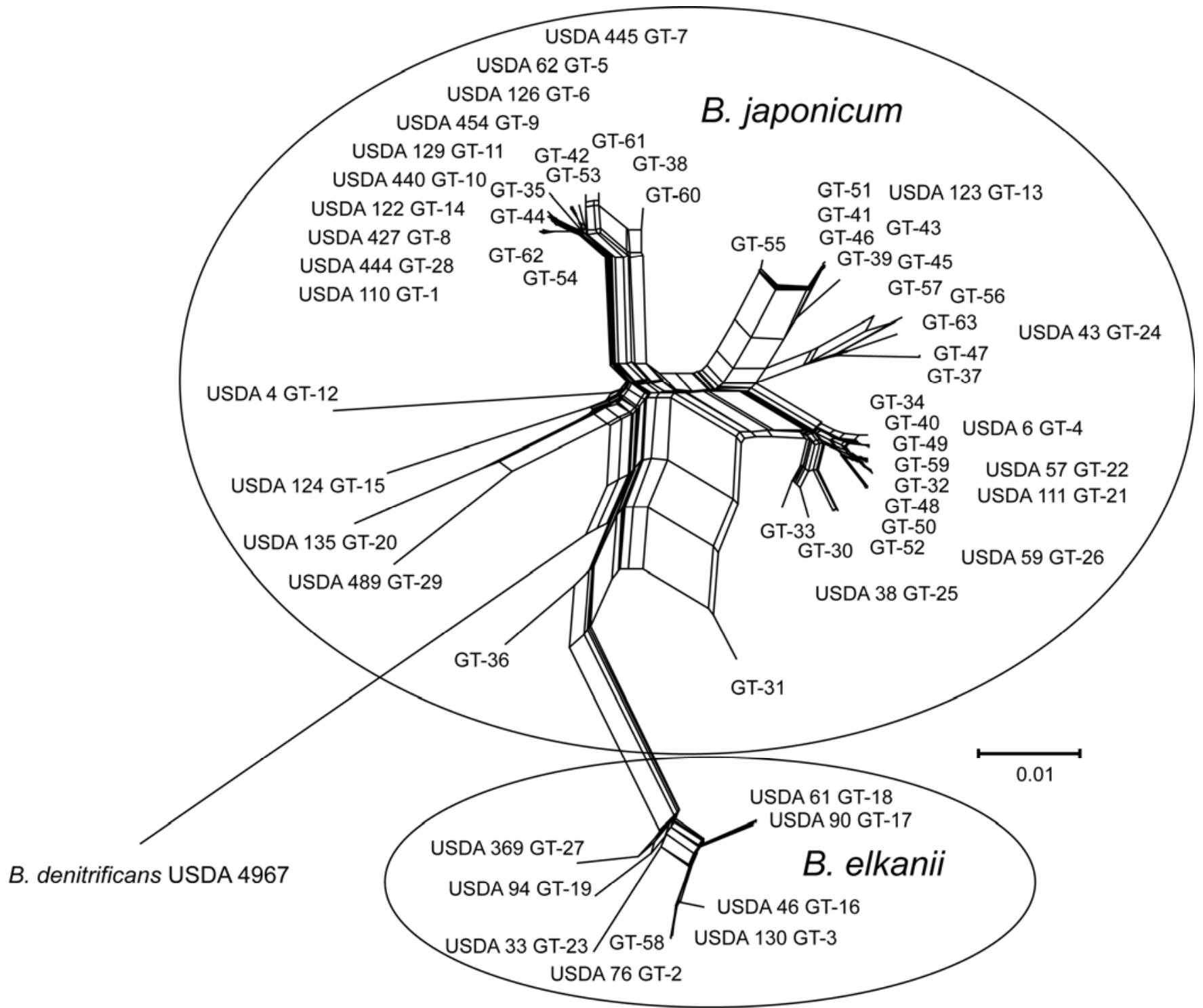

Fig. 3. Estimated evolutionary relationships among the bacteroid genotypes that occupied nodules of uninoculated soybean (Glycine max L. Merr) Roundupready NK Brand S49-Q9 and culture collection soybean strains. Sequences of seven chromosomal loci were contactenated to produce an alignment of 2,960 bp used in Splitstree version 4.10 (Huson and Bryant 2006) to display evolutionary relationships of the chromosomes as the network algorithm NeighborNet (Bryant and Moulton 2004). A significant probability for recombination among the various chromosomes was shown $(P$ value $=0.0)$ using the pairwise homoplasy index test (Bruen et al. 2006). Two circles surround the genotypes that were identified as those of Bradyrhizobium japonicum and B. elkanii. Results with B. denitrificans United States Department of Agriculture (USDA) 4967 were used as outgroup to the network. 
GT among members of the genus Bradyrhizobium originating from symbioses with other legume hosts in different geographic locations.

\section{MATERIALS AND METHODS}

Strains of Bradyrhizobium used in MLST analysis.

A selection of strains originating from several different legume hosts (Table 3) was analyzed to demonstrate that the MLST method developed could be applied to all members within the genus Bradyrhizobium. The analysis also included the named species B. japonicum 3I1b6, USDA 6 or ATCC 10324 (Jordan 1982), B. japonicum USDA 110 (Date and Decker 1965), B. elkanii USDA 76 (Kuykendall et al. 1992), B. denitrificans USDA 4967 (van Berkum et al. 2006), B. canariense LMG22265 (Vinuesa et al. 2005), B. yuanmingense LMG21827 (Yao et al. 2002), B. iriomotense LMG24129 (Islam et al. 2008), B. pachyrhizi PAC48 (Ramírez-Bahena et al. 2009), B. jicamae PAC68 (Ramírez-Bahena et al. 2009), and $B$. betae PL7HG (Rivas et al. 2004). Variation in the GT of $B$. japonicum and B. elkanii, all isolated from soybean, were determined with 190 strains in the USDA-ARS Rhizobium Germplasm Resource Collection (Table 2).

\section{Cell culture, soybean source, DNA extraction}

from cultured cells, and bacteroids.

Cultures of Bradyrhizobium spp. were recovered from lyophilized storage in modified arabinose-gluconate (MAG) broth (van Berkum 1990) and allowed to grow at $30^{\circ} \mathrm{C}$ for 7 days. These broth cultures were plated on solid MAG medium and incubated at $30^{\circ} \mathrm{C}$ for 5 days. Several single colonies of each culture were suspended in 200- $\mu$ l volumes of Prepman Ultra (Applied Biosystems, Foster, CA, U.S.A.) that had been placed in clear, boil-proof microcentrifuge tubes (Labsource, Chicago). PCR-quality DNA was then extracted according to the manufacturer's specifications.

Uninoculated soybean (G. $\max$ L. Merr) Roundup-ready NK Brand S49-Q9 had been sown for crop production in the South Farm of the Beltsville Agricultural Research Center during summer 2008. Plants at the R1 and R3 growth stages were randomly chosen at different locations throughout the field and were carefully dug up to collect as much of the root systems as possible. All of the nodules from five plants were gathered and separated into two collections according to their location on the tap or the lateral roots. Each nodule was then placed in separate microcentrifuge tubes followed by the addition of 0.5 $\mathrm{ml}$ of deionized water. The nodules in each tube were then crushed with clean pestles for $1.5-\mathrm{ml}$ microcentrifuge tubes to release the bacteroids. The debris of the nodules was allowed to settle for a few minutes before drawing off the bacteroid suspensions, which were placed into boil-proof microcentrifuge tubes. The bacteroids were collected at 14,000 rpm for 5 min, the supernatant water was discarded, and $200 \mu \mathrm{l}$ of Prepman Ultra was added to extract PCR-quality DNA as described before.

\section{Development of MLST analysis.}

The complete genome sequence of B. japonicum USDA 110 (Kaneko et al. 2002) was used to select seven chromosomal loci (Table 1) for the development of MLST. Selection of the loci was based on the condition that they were named genes with wide distribution across the chromosome. The loci chosen were aligned with the same loci obtained from the complete genome sequence of Bradyrhizobium sp. strain BTAil (Giraud et al. 2007) in search of conserved regions for the selection of PCR and sequence primers using the primer design software package Oligo Primer Analysis software (version 6.65; Molecu- lar Biology Insights, Inc., Cascade, CO, U.S.A.). The oligonucleotides selected (Table 1) with Oligo were synthesized by Sigma-Genosys (The Woodlands, TX, U.S.A.) and were received as dried preparations. Upon receipt, the primers were dissolved with $10 \mathrm{mM}$ Tris buffer, $\mathrm{pH} 8.0$, to a final concentration of $1,000 \mathrm{pmol}$ and were stored at $-20^{\circ} \mathrm{C}$. The PCR reactions for each locus were then optimized by using the FailSafe PCR PreMix selection kit (Epicenter) and the thermal cycle protocol described by van Berkum and Fuhrmann (2000) with an MJ Research PTC-225 Peltier thermal cycler (MJ Research Inc., Waltham, MA, U.S.A.) using genomic DNA of both B. japonicum USDA 110 and $B$. elkanii USDA 76 as templates. These PCR reactions were analyzed by horizontal agarose gel electrophoresis to select the FailSafe PCR system (Epicenter) determined to be the most suitable for PCR amplification with the DNA preparations of the strains and bacteroids used in this investigation. The presence of a single PCR product of the expected molecular size for each primer pair using each of the different templates was verified before sequence analysis. Each PCR product was then purified, especially to remove the PCR primers, by using the Ampure PCR purification system (Agincourt Bioscience Corporation, Beverly, MA, U.S.A.). The purified PCR products were used in two or sometimes four sequence reactions each with one of the nested sequence primers or in combination with the original PCR primers. An Applied Biosystems 3130 DNA Analyzer in combination with a Dye Deoxy Terminator cycle sequencing kit (Applied Biosystems) was used for sequencing the purified PCR products as described previously (van Berkum et al. 1996).

\section{Data analysis.}

A Microsoft Access database was created to compile the data. The strategy for design and manipulation of the database was as described by van Berkum and associates (2006). The sequence length entered for each locus was identical and the same alleles were identified using the software Sequence Comparator version 2.0.1 written by K. Jolley (Oxford). As additional alleles were identified, they were assigned different allele numbers in the database. In each case, allelic variation was verified by confirming the sequence disparity using Genedoc (version 2.6.001) and then checking the electropherograms produced by the sequence analysis to substantiate differences. In the case of ambiguities, the sequence analysis was repeated.

The relationships function in Microsoft Access was used to create a query of the allelic allocation of the seven loci for each of the 190 soybean strains. The resulting tabulated data were exported as a Microsoft Excel file, which was converted and then modified as a text input for a Pearl Script to obtain a list of GT (Table 2). A similar input file was created with the bacteroid results. However, the bacteroid and culture collection data were combined before deriving the list of GT. The GT of the bacteroid samples alone were subsequently derived by removing the culture collection strains. The data obtained with the bacteroids were then divided into GT that occupied the tap or lateral root nodules. These two lists were used to create a histogram with Lotus Freelance Graphics (Lotus Development Corporation, Cambridge, MA, U.S.A.) that portrayed the tap and lateral root nodule occupancy of the five soybean plants grown in the South Farm of the Beltsville Agricultural Research Center.

Sequences of the seven loci also were concatenated using the relationships function in Microsoft Access. Alignments obtained of the concatenated sequences were used to investigate the relationships among chromosomes of the soybean strains and the Bradyrhizobium cultures that originated from different legume host plants and that also included the different proposed species within the genus. In addition, alignments of concate- 
nated sequences were used to investigate the relationships among chromosomes of the soybean culture collection strains and those of the nodule occupants. Fasta files of the alignments were analyzed with SplitsTree version 4.10 (Huson and Bryant 2006) to display evolutionary relationships of the chromosomes as the network algorithm NeighborNet (Bryant and Moulton 2004). The alignment of the soybean strains and the Bradyrhizobium cultures that originated from different legume host plants also were used to test for recombination using the pairwise homoplasy index $\left(\Phi_{\mathrm{w}}\right.$ statistic) of Bruen and associates (2006).

\section{ACKNOWLEDGMENTS}

We thank C. Vereecke, BCCM/LMG Bacteria Collection, Ghent, Belgium for providing B. canariense LMG22265, B. iriomotense LMG24129, and B. yuanmingense LMG21827; E. Velázquez, Departamento de Microbiología y Genética, Universidad de Salamanca, Spain for B. pachyrhizi PAC48, B. jicamae PAC68, and B. betae PL7HG; E. Martínez-Romero, The Center for Genomic Sciences of the National Autonomous University of Mexico for the lupin strains F13 and CICS70; and J. G. Howieson, Centre for Rhizobium Studies, School of Biological Sciences and Biotechnology, Murdoch University, Australia for CB564.

\section{LITERATURE CITED}

Alazard, D. 1985. Stem and root nodulation in Aeschynomene spp. Appl. Environ. Microbiol. 50:732-734.

Berg, R. K., Loynachan, T. E., Zablotowicz, R. M., and Lieberman, M. T. 1988. Nodule occupancy by introduced Bradyrhizobium japonicum in Iowa soils. Agron. J. 80:876-881.

Boonkerd, N., Weber, D. F., and Bezdicek, D. F. 1978. Influence of Rhizobium japonicum strains and inoculation methods on soybeans grown in rhizobia-populated soils. Agron. J. 70:547-549.

Brooks, W. P. 1901. Agriculture, vol. II. Manures, Fertilizers and Farm Crops, Including Green Manuring and Crop Rotation. The Home Correspondence School, Springfield, MA, U.S.A.

Bruen, T. C., Philippe, H., and Bryant D. 2006. A simple and robust statistical test for detecting the presence of recombination. Genetics 172:2665-2681.

Bryant, D., and Moulton V. 2004. Neighbor-Net: An agglomerative method for the construction of phylogenetic networks. Mol. Biol. Evol. $21: 255-265$

Caldwell, B. E. 1969. Initial competition of root-nodule bacteria on soybeans in a field environment. Agron. J. 61:813-815.

Caldwell, B. E., and Hartwig, E. E. 1970. Serological distribution of soybean root nodule bacteria in soils of southeastern USA. Agron. J. 62:621-622.

Caldwell, B. E., and Vest, G. 1968. Nodulation interactions between soybean genotypes and serogroups of Rhizobium japonicum. Crop Sci. 8:680-682.

Caldwell, B. E., and Weber, D. F. 1970. Distribution of Rhizobium japonicum serogroups in soybean nodules affected by planting dates. Agron. J. 62:12-14.

Carroll, W. R. 1934. A study of Rhizobium species in relation to nodule formation on the roots of Florida legumes: I. Soil Sci. 37:117-135.

Cotrell, H. M., Otis, D. H., and J. G. Haney. 1900. Soil inoculation for soy beans. Kans. Agric. Exp. Stn. Bull. 96:97-116.

Cregan, P. B., and Keyser, H. H. 1986. Host restriction of nodulation by Bradyrhizobium japonicum strain USDA 123 in soybean. Crop Sci. 26:911-916.

Damirgi, S. M., Frederick, L. R., and Anderson, I. C. 1967. Serogroups of Rhizobium japonicum in soybean nodules as affected by soil types. Agron. J. 59:10-12.

Date, R. A., and Decker, A. M. 1965. Minimal antigenic constitution of 28 strains of Rhizobium japonicum. Can. J. Microbiol. 11:1-8.

Eardly, B. D., and van Berkum, P. 2005. Use of population genetic structure to define species limits in Rhizobiacea. Symbiosis 38:109-122.

Ellis, W. R., Ham, G. E., and Schmidt, E. L. 1984. Persistence and recovery of Rhizobium japonicum inoculum in a field soil. Agron. J. 76:573-576.

Feil, E. J. 2004. How stable are the core genes of bacterial pathogens? ASM News 6:234-238.

Fred, E. B., Baldwin, I. L., and McCoy, E. 1932. Some factors which influence the growth and longevity of the nodule bacteria. Pages 104-145 in: Root Nodule Bacteria and Leguminous Plants, vol. 5. I. L. Baldwin, E. McCoy, and E. B. Fred, eds. University of Wisconsin, Madison.
Fuhrmann, J. J. 1990. Symbiotic effectiveness of indigenous soybean bradyrhizobia as related to serological, morphological, rhizobitoxine, and hydrogenase phenotypes. Appl. Environ. Microbiol. 56:224-229.

Giraud, E., Moulin, L., Vallenet, D., Barbe, V., Cytryn, E., Avarre, J. C. Jaubert, M., Simon, D., Cartieaux, F., Prin, Y., Bena, G., Hannibal, L., Fardoux, J., Kojadinovic, M., Vuillet, L., Lajus, A., Cruveiller, S., Rouy, Z., Mangenot, S., Segurens, B., Dossat, C., Franck, W. L., Chang, W. S., Saunders, E., Bruce, D., Richardson, P., Normand, P., Dreyfus, B., Pignol, D., Stacey, G., Emerich, D., Verméglio, A., Médigue, C., and Sadowsky, M. 2007. Legumes symbioses: Absence of nod genes in photosynthetic bradyrhizobia. Science 316:1307-1312.

Ham, G. E., Caldwell, V. B., and Johnson, H. W. 1971. Evaluation of Rhizobium japonicum inoculants in soils containing naturalized populations of rhizobia. Agron. J. 63:301-303.

Hopkins, C. G. 1904. Nitrogen bacteria and legumes. Ill. Agric. Exp. Stn. Bull. 94:307-328.

Huson, D. H., and Bryant D. 2006. Application of phylogenetic networks in evolutionary studies. Mol. Biol. Evol. 23:254-267.

Islam M. S., Kawasaki H., Muramatsu Y., Nakagawa Y., and Seki, T. 2008. Bradyrhizobium iriomotense sp. nov., isolated from a tumor-like root of the legume Entada koshunensis from Iriomote Island in Japan. Biosci. Biotechnol. Biochem. 72:1416-1429.

Johnson, H. W., Means, U. M., and Weber, C. R. 1965. Competition for nodule sites between strains of Rhizobium japonicum applied as inoculum and strains in the soil. Agron. J. 57:179-185.

Jordan, D. C. 1982. Transfer of Rhizobium japonicum Buchanan 1980 to Bradyrhizobium gen. nov., a genus of slow-growing root nodule bacteria from leguminous plants. Int. J. Syst. Bacteriol. 32:136-139.

Kaneko, T., Nakamura, Y., Sato, S., Minamisawa, K., Uchiumi, T., Sasamoto, S., Watanabe, A., Idesawa, K., Iriguchi, M., Kawashima, K., Kohara, M., Matsumoto, M., Shimpo, S., Tsuruoka, H., Wada, T., Yamada, M., and Tabata, S. 2002. Complete genomic sequence of nitrogen-fixing symbiotic bacterium Bradyrhizobium japonicum USDA 110. DNA Res. 9:189-197.

Kapusta, G., and Rouwenhorst, D. L. 1973. Influence of inoculum size on Rhizobium japonicum serogroup distribution frequency in soybean nodules. Agron. J. 65:916-919.

Keyser, H. H., and Cregan, P. B. 1987. Nodulation and competition for nodulation of selected soybean genotypes among Bradyrhizobium japonicum serogroup 123 isolates. Appl. Environ. Microbiol. 53:26312635.

Kuykendall, L. D., Saxena, B., Devine, T. E., and Udell, S. E. 1992. Genetic diversity in Bradyrhizobium japonicum Jordan 1982, and a proposal for Bradyrhizobium elkanii sp. nov. Can. J. Microbiol. 38:501-505.

Kvien, C. S., Ham, G. E., and Lambert, J. W. 1981. Recovery of introduced Rhizobium japonicum strains by soybean genotypes. Agron. J. 73:900-905.

Leonard, L. T. 1923. Nodule-production kinship between the soy bean and the cowpea. Soil Sci. 15:277-283.

Lindemann, W. C., Schmidt, E. L., and Ham, G. E. 1974. Evidence for double infection within soybean nodules. Soil Sci. 118:274-279.

Maiden, M. C. J., Bygraves, J. A., Feil, E., Morelli, G., Russell, J. E., Urwin, R., Zhang, Q., Zhou, J., Zurth, K., Caugant, D. A., Feavers, I. M., Achtman, M., and Spratt, B. G. 1998. Multilocus sequence typing: A portable approach to the identification of clones within populations of pathogenic microorganisms. Proc. Natl. Acad. Sci. U.S.A. 95:31403145 .

Means, U. M., Johnson, H. W., and Date, R. E. 1964. Quick serological method of classifying strains of Rhizobium japonicum in nodules. J. Bacteriol. 87:547-553.

Moawad, M., and Schmidt E. L. 1987. Occurrence and nature of mixed infections in nodules of field-grown soybeans Glycine max. Biol. Fertil. Soils 5:112-114.

Mpepereki, S., and Wollum, A. G. 1991. Diversity of indigenous Bradyrhizobium japonicum in North Carolina soils. Biol. Fertil. Soils. 11:121127.

Norris, D. O. 1959. The role of calcium and magnesium in the nutrition of Rhizobium. Aust. J. Agric. Res. 10:651-698.

Ramírez-Bahena, M. H., Peix, A., Rivas, R., Camacho, M., RodríguezNavarro, D. N., Mateos, P. F., Martinez-Molina, E., Willems, A., and Velázquez, E. 2009. Bradyrhizobium pachyrhizi sp. nov. and Bradyrhizobium jicamae sp. nov., isolated from effective nodules of Pachyrhizus erosus. Int. J. Syst. Evol. Microbiol. 59:1929-1934.

Rivas, R., Willems, A., Luis Palomo, J., Garcia-Benavides, P., Mateos, P. F., Martínez-Molina, E., Gillis, M., and Velázquez, E. 2004. Bradyrhizobium betae sp. nov., isolated from roots of Beta vulgaris affected by tumour-like deformations. Int. J. Syst. Evol. Microbiol. 54:12711275.

Schmidt, E. L., Bankole, R. O., and Bohlool, B. B. 1968. Fluorescentantibody approach to study of rhizobia in soil. J. Bacteriol. 95:1987-1992. 
Schmidt, E. L., Zidwick, M. J., and Abebe, H. M. 1986. Bradyrhizobium japonicum serocluster 123 and diversity among member isolates. Appl. Environ. Microbiol. 51:1212-1215.

Sears, O. H., and Carroll, W. R. 1927. Cross inoculation with cowpea and soybean nodule bacteria. Soil Sci. 24:413-419.

Thompson, J. A., Bhromsiri, A., Shutsrirung, A., and Lillakan, S. 1991. Native root-nodule bacteria of traditional soybean-growing areas of northern Thailand. Plant Soil 135:53-65.

van Berkum, P. 1990. Evidence for a third uptake hydrogenase phenotype among the soybean bradyrhizobia. Appl. Environ. Microbiol. 56:38353841 .

van Berkum, P., and Eardly, B. D. 2005. Impact of genomics on the reconstruction of evolutionary relationships of nitrogen-fixing bacteria and implications for taxonomy. Pages 201-219 in: Genomes and Genomics of Nitrogen-fixing Organisms. R. Palacios and W. E. Newton, eds. Springer, The Netherlands.

van Berkum, P., and Fuhrmann, J. J. 2000. Evolutionary relationships among the soybean bradyrhizobia reconstructed from 16S rRNA gene and internally transcribed spacer region sequence divergence. Int. J. Syst. Evol. Microbiol. 50:2165-2172.

van Berkum, P., and Fuhrmann, J. J. 2009. Evidence from Internally Transcribed Spacer sequence analysis of soybean strains that extant Bradyrhizobium spp. are likely the products of reticulate evolutionary events. Appl. Environ. Microbiol. 75:78-82.

van Berkum, P., Elia, P., and Eardly, B. D. 2006. Multilocus Sequence Typing as an approach for population analysis of Medicago-nodulating rhizobia. J. Bacteriol. 188:5570-5577.

van Berkum, P., Badri, Y., Elia, P., Aouani, M. E., and Eardly, B. D. 2007. Chromosomal and symbiotic relationships of rhizobia nodulating Medicago truncatula and M. laciniata. Appl. Environ. Microbiol. 73:75977604.

van Berkum, P., Elia, P., and Eardly, B. D. 2010. Application of Multilocus
Sequence Typing to study the genetic structure of megaplasmids in Medicago-nodulating rhizobia. Appl. Environ. Microbiol. 76:3967-3977.

Vest, G., Caldwell, B. E., and Petersen, H. D. 1971. Variability associated with sampling of Rhizobium japonicum populations in soybeans. Crop Sci. 11:780-782.

Vinuesa, P., León-Barrios, M., Silva, C., Willems, A., Jarabo-Lorenzo, A., Pérez-Galdona, R., Werner, D., and Martínez-Romero, E. 2005. Bradyrhizobium canariense sp. nov., an acid-tolerant endosymbiont that nodulates endemic genistoid legumes (Papilionoideae: Genisteae) from the Canary Islands, along with Bradyrhizobium japonicum bv. genistearum, Bradyrhizobium genospecies alpha and Bradyrhizobium genospecies beta. . Int. J. Syst. Evol. Microbiol. 55:569-575.

Weber, D. F., Keyser, H. H., and Uratsu, S. L. 1989. Serological distribution of Bradyrhizobium japonicum from U.S. soybean production areas. Agron. J. 81:786-789.

Yao, Z. Y., Kan, F. L., Wang, E. T., Wei, G. H., and Chen, W. X. 2002. Characterization of rhizobia that nodulate legume species of the genus Lespedeza and description of Bradyrhizobium yuanmingense sp. nov. Int. J. Syst. Evol. Microbiol. 52:2219-2230.

Yokoyama, T., Ando, S., and Tsuchiya, K. 1999. Serological properties and antibiotic resistance of soybean bradyrhizobiaisolated from Thailand. Soil Sci. Plant Nutr. 45:505-515.

\section{AUTHOR-RECOMMENDED INTERNET RESOURCES}

United States Department of Agriculture, Economic Resource Service websites for information on soybean: www.ers.usda.gov/Briefing/SoybeansOilcrops www.ers.usda.gov/briefing/soybeansoilcrops/2010_19baseline.htm www.ers.usda.gov/Briefing/SoybeansOilcrops/background.htm

GeneDoc: www.nrbsc.org/gfx/genedoc/index.html 